\title{
Anti-CD3 antibody therapy for type 1 diabetes mellitus: outcome 4 years after treatment
}

A short course of anti-CD3 antibody therapy decreases the insulin needs of patients with type 1 diabetes mellitus (T1DM) during the first few years after diagnosis, new data of a phase II trial reveal; the effects of the antibody therapy are influenced by age and residual $\beta$-cell mass at initiation of treatment.

Anti-CD3 antibody therapy aims to restore immune tolerance to target autoantigens in patients with T1DM without affecting their responses to other antigens. In a phase II, randomized, double-blind, placebo-controlled trial of a 6-day treatment with humanized antiCD3 antibody, residual $\beta$-cell function was preserved and insulin requirements reduced for up to 18 months. Now, the researchers report treatment outcomes 4 years after the original therapy.

In the initial trial, the researchers randomly allocated 80 patients (aged 12-39 years) with new onset of T1DM to receive humanized anti-CD3 antibody treatment or placebo. All participants received intensive insulin therapy throughout the trial.

\section{Our data represent a step} towards treatment of T1DM at an early stage... 77

Over the 48 months, insulin requirements were reduced in patients who received the antibody therapy compared with those of the placebo group. The difference in insulin requirements between the groups resulted from a decline in residual $\beta$-cell function in the placebo group but maintenance of this function for at least 24 months in antibody-treated individuals. This apparent suppression of $\beta$-cell destruction by the antibody therapy thereafter waned.

Treatment effects correlated with age and residual $\beta$-cell mass at initiation of treatment. In a subgroup analysis, the metabolic control of younger (12-27 years) but not older (27-39 years) individuals benefited from the therapy. Furthermore, patients with early stages of $\beta$-cell destruction benefited most from the therapy. Although no long-term adverse effects of the therapy were reported, transient, flu-like symptoms were noted during and shortly after the treatment.

"Our data represent a step towards treatment of T1DM at an early stage, and possibly towards its prevention," comments senior researcher Daniel Pipeleers of Brussels Free University. "It will now be necessary to define efficacious and safe protocols for antibody administration in patients with recentonset of T1DM, including children, as well as in individuals at high risk for this disease."

\section{Carol Wilson}

Original article Keymeulen, B. et al. Four-year metabolic outcome of a randomised controlled CD3-antibody trial in recent-onset type 1 diabetic patients depends on their age and baseline residual beta cell mass. Diabetologia 53 614-623 (2010) 